\title{
TUNNELING TRANSITIONS IN DE SITTER SPACETIME
}

\author{
BUM-HOON LEE \\ Department of Physics and Center for Quantum Spacetime, Sogang University \\ Seoul 121-742, Korea \\ bhl@sogang.ac.kr \\ WONWOO LEE \\ Center for Quantum Spacetime, Sogang University \\ Seoul 121-742, Korea \\ warrior@sogang.ac.kr
}

\begin{abstract}
We study tunneling transitions in de Sitter space. The self-gravitating scalar field with $O(4)$ symmetry is taken into account. In this consideration the inherent property of Euclidean de Sitter geometry plays an important role in vacuum tunneling transitions. We extend Parke's results to other classes of true vacuum bubbles, false vacuum bubbles, and degenerate cases. We mention that the pair creation of black holes can be possible in the background of bubble solutions. This article is prepared for the proceedings of International Symposium on Cosmology and Particle Astrophysics(CosPA2008) in Korea, Oct 2008 .
\end{abstract}

Keywords: Bubble nucleation; extension of Parke's results; black hole pair creation.

\section{Introduction}

The vacuum tunneling transition of a self-gravitating scalar field in de Sitter spacetime has been considered in many cosmological scenarios with great interest. The first and important one was so-called old inflationary universe scenario, solving the horizon and flatness problems of standard cosmology but leaving the graceful exit problem, in Refs. 1,2,3. The problem was solved by new inflationary scenario ${ }^{4,5}$ even if the problem about the initial value of the inflaton was remained. Hawking and Moss $^{6}$ proposed another version of the inflationary scenario, which describes the scalar field jumping simultaneously at the top of the potential barrier. In addition, it was pointed out that one of the bubbles could be our universe as a standard open model. ${ }^{7,8}$ Another approach for resolving the graceful exit problem was done in Brans-Dicke theory of gravity. ${ }^{9}$ An open universe scenario from inflation was also presented through combining old inflation with new inflation. ${ }^{10}$ The string theory landscape paradigm has many stable and metastable vacua. ${ }^{11,12}$ So the tunneling transition becomes a remarkable event in this framework as well as eternal inflation. ${ }^{13,14,15}$ The eternal inflation is in the center of the multiverse cosmological paradigm although inflationary spacetimes have the problem of incompleteness in 
past directions. ${ }^{16}$ Multiverses or parallel universes are classified into four levels by Tegmark. ${ }^{17}$ Level II multiverse in his four-level hierarchy is related to post-inflation bubbles.

Actually, the formation of a true vacuum bubble via the decay of a metastable vacuum at zero temperature in flat space was considered in Ref. 18 and developed in Ref. 19. The process in the presence of gravity was studied in Ref. 20. This result for the formation of the small true vacuum bubble within the large false vacuum background was enlarged by Parke ${ }^{21}$ to the case with arbitrary cosmological constants. An extension of this theory to the case of non-zero temperatures has been found by Linde ${ }^{22}$ in the absence of gravity. The effect of the Gauss-Bonnet term on vacuum decay was also studied. ${ }^{23}$ Marvel and Wesley studied thin-wall instanton with negative tension wall and its relation to Witten's bubble of nothing. ${ }^{24}$ In Ref. 25, the authors discussed the possible four different instantons in Euclidean de Sitter space. The authors concluded that one class of solutions is possible. Brown and Weinberg studied thermal derivation of the Coleman-De Luccia tunneling. ${ }^{26}$ The authors clarified the meaning of the bounce solution itself as well as oscillating bounce solutions. ${ }^{27}$ Hawking-Moss transition was analyzed as a thermal fluctuation. ${ }^{28}$ The tunneling in de Sitter space may be interpreted as a finite temperature effect due to the Gibbons-Hawking temperature. ${ }^{29}$

As for the false vacuum bubble, Lee and Weinberg ${ }^{30}$ obtained the method for the nucleation of a false vacuum bubble in de Sitter space. The authors obtained the ratio between the decay rate of the true vacuum and that of the false vacuum. However, the nucleation rate and the radius of a false vacuum bubble were not obtained. In addition, the nucleation and evolution of bubbles were investigated in the model with $\mathrm{O}(3)$-symmetric scalar field coupled to gravity in the high temperature limit. ${ }^{31}$ In their work, a false vacuum region like a global monopole remains at the center of a bubble. It was obtained the mechanism for false vacuum bubble nucleation within the true vacuum background with arbitrary cosmological constants in the Einstein theory of gravity with a nonminimally coupled scalar field. ${ }^{32,33}$

Next, we will consider black hole pair creation. The vacuum of a strong field can decay due to the Schwinger mechanism ${ }^{34}$ for particle production. Particle production is one of the decaying methods of the background field or given vacuum energy (for recent works, see Ref. 35). For the black hole creation, Gross, Perry, and Yaffe $^{36}$ obtained the nucleation rate for black holes in a thermal bath. The nucleation of black holes in the de Sitter universe with a semiclassical instability was considered by Ginsparg and Perry. ${ }^{37}$ Caldwell et al. studied black hole pair creation in the presence of a domain wall ${ }^{38}$ using the cut-and-paste procedure, where the background has vanishing cosmological constant and the probability was obtained. The repulsive property of the domain wall give rise to black hole pair creation.

Recently, we have investigated the possible types of the nucleation of vacuum bubbles. ${ }^{39}$ We classified true vacuum bubbles in de Sitter background and present some numerical solutions. The nucleation rate and the radius of true and false 
vacuum bubbles are analytically computed using the thin-wall approximation. We obtained static bubble wall solutions of junction equation with black hole pair. In this article, we will summarize our results and speculate about the implication in the multiverse scenario. Tunneling transitions between the degenerate vacua in flat and anti-de Sitter space are also studied in Ref. 40.

\section{Bubble Nucleation and Pair Production of Black Holes}

The bubble nucleation rate or the decay rate of background vacuum is semiclassically given by

$$
\Gamma / V=A e^{-B / \hbar},
$$

where $B$ is the difference between Euclidean action corresponding to bubble solution and that of the background. The pre-factor $A$ is discussed in Ref. 41, that with some gravitational corrections in Ref. 42. We are interested in finding the coefficient $B$.

The Euclidean field equations for $\Phi$ and $\rho$ have the form

$$
\begin{aligned}
& \Phi^{\prime \prime}+\frac{3 \rho^{\prime}}{\rho} \Phi^{\prime}=\frac{d U}{d \Phi}, \\
& \rho^{\prime \prime}=-\frac{\kappa}{3} \rho\left(\Phi^{\prime 2}+U\right),
\end{aligned}
$$

respectively and the Hamiltonian constraint is given by

$$
\rho^{\prime 2}=1+\frac{\kappa \rho^{2}}{3}\left(\frac{1}{2} \Phi^{\prime 2}-U\right)
$$

We consider only the case with initial de Sitter space. The boundary conditions for the bounce are

$$
\left.\frac{d \Phi}{d \eta}\right|_{\eta=0}=0,\left.\quad \frac{d \Phi}{d \eta}\right|_{\eta=\eta_{\max }}=0, \quad \rho_{\eta=0}=0, \text { and } \rho_{\eta=\eta_{\max }}=0,
$$

where $\eta_{\max }$ is a finite value in Euclidean de Sitter space. The asymptotic value of $\Phi(\eta)$ is given by

$$
\lim _{\eta \rightarrow \eta_{\max }} \Phi(\eta) \simeq \Phi_{F / T},
$$

where $\Phi_{F}$ is for a true vacuum bubble nucleation and $\Phi_{T}$ is for a false one. Because of the finiteness of $\eta_{\max }, \Phi\left(\eta_{\max }\right)$ is exponentially approaching to but not reaching $\Phi_{F / T}$.

Now, we assume the thin-wall approximation scheme to evaluate $B$. The Euclidean action can be divided into three parts :

$$
B=B_{\text {in }}+B_{\text {wall }}+B_{\text {out }},
$$

where the configuration of the outside of the wall will be zero.

With this assumption we can classify true vacuum bubbles into nine types and false vacuum bubbles into three types. These true vacuum bubbles correspond to the small flat bubble with large dS background, the small AdS bubble with large 


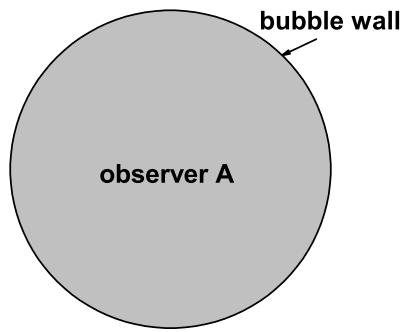

(a) the inside observer's point of view

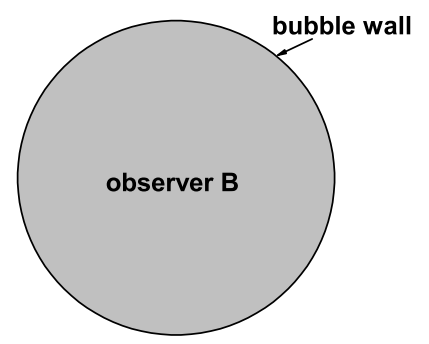

(b) the outside observer's point of view

Fig. 1. Two observers's point of view are illustrated. The left figure, (a), represents the inside observer's point of view. The right figure, (b), represents the outside observer's point of view. Every observers see themselves surrounded by the wall after the transition. Thus, every observers remain inside the wall.

dS background, the small dS bubble with large dS background, the half flat bubble with half dS background, the half AdS bubble with half dS background, the half dS bubble with half dS background, the large flat bubble with small dS background, the large AdS bubble with small dS background, and the large dS bubble with small dS background. Those false vacuum bubbles correspond to Lee-Weinberg transition, the reflected version of the half dS bubble with half dS background, and the reflected version of the large dS bubble with small dS background.

Now, we will consider the transition of degenerate vacua in de Sitter space. It is possible that the tunneling occurs via the potential with degenerate vacua in de Sitter space. The numerical solution of this tunneling was obtained in Ref. 27. The solution of $\Phi(\eta)$ is only seen in their paper. This tunneling transition is possible due to the changing role of the second term in Eq. (2) from damping to accelerating during the transition.

The situation after this tunneling transition is different from an ordinary case of a true vacuum bubble. Because of the four-sphere of Euclidean de Sitter space, each observer, before the tunneling transition, sees also himself/herself surrounded by the wall after the transition. Thus, every observers remain inside the wall. The schematic diagrams for this situation are illustrated in (a) and (b) of Fig. 1. Tunneling transitions between the degenerate vacua in flat and anti-de Sitter space are recently studied in Ref. 40. This situation is similar to the creation of RandallSundrum type configuration if the mechanism is applied in high-dimensional theory.

Caldwell et al. studied black hole pair creation in the presence of a domain wall ${ }^{38}$ using the cut-and-paste procedure, where the background has vanishing cosmological constant. The pair production of black holes is possible in the presence of our bubble solutions. Furthermore, the geometry for the creation can be made more naturally. The creation rate was obtained in Ref. 39. 


\section{Discussion}

We have studied tunneling transition in de Sitter space. We have obtained some numerical solutions and analytic computations for a bubble nucleation. We have considered black hole pair creation in the presence of the bubble wall.

After the tunneling transition in cases of degenerate vacua, every observers see themselves surrounded by the wall. Thus, every one remains inside the wall. This situation is different from one of the ordinary nucleation of a vacuum bubble.

The transition including a bubble nucleation and black hole pair creation give the lore to understand the mechanism how the complicated spacetime structure could be formed in the very early universe and multiverse scenario.

\section{Acknowledgments}

We would like to thank Sang Pyo Kim for his hospitality at International Symposium on Cosmology and Particle Astrophysics (CosPA2008) in Pohang, Korea, 29-31 Oct 2008. This work was supported by the Korea Science and Engineering Foundation (KOSEF) grant funded by the Korea government(MEST) through the Center for Quantum Spacetime(CQUeST) of Sogang University with grant number R11 - 2005 - 021. W.L. was supported by the Korea Research Foundation Grant funded by the Korean Government(MOEHRD)(KRF-2007-355-C00014).

\section{References}

1. A. H. Guth, Phys. Rev. D 23, 347 (1981).

2. K. Sato, Mon. Not. R. Astron. Soc. 195, 467 (1981).

3. A. H. Guth and E. J. Weinberg, Nucl. Phys. B 212, 321 (1983).

4. A. D. Linde, Phys. Lett. B 108, 389 (1982).

5. A. Albrecht and P. J. Steinhardt, Phys. Rev. Lett. 48, 1220 (1982).

6. S. W. Hawking and I. G. Moss, Phys. Lett. B 110, 35 (1982).

7. J. R. Gott, Nature 295, 304 (1982).

8. J. R. Gott and T. S. Statler, Phys. Lett. B 136, 157 (1984).

9. D. La and P. J. Steinhardt, Phys. Rev. Lett. 62, 376 (1989); ibid. 62, 1066(E) (1989).

10. M. Bucher, A. S. Goldhaber and N. Turok, Phys. Rev. D 52, 3314 (1995).

11. L. Susskind, hep-th/0302219.

12. S. Kachru, R. Kallosh, A. Linde and S. P. Trivedi, Phys. Rev. D 68, 046005 (2003).

13. A. Vilenkin, Phys. Rev. D 27, 2848 (1983).

14. A. D. Linde, Phys. Lett. B 175, 395 (1986).

15. A. H. Guth, Phys. Rep. 333, 555 (2000).

16. A. Borde, A. H. Guth and A. Vilenkin, Phys. Rev. Lett. 90, 151301 (2003).

17. M. Tegmark, in Universe or Multiverse? ed. B. Carr (Cambridge, 2007).

18. M. B. Voloshin, I. Yu. Kobzarev and L. B. Okun, Yad. Fiz. 20, 1229 (1974) [Sov. J. Nucl. Phys. 20, 644 (1975)].

19. S. Coleman, Phys. Rev. D 15, 2929 (1977); ibid. D 16, 1248(E) (1977).

20. S. Coleman and F. De Luccia, Phys. Rev. D 21, 3305 (1980).

21. S. Parke, Phys. Lett. B 121, 313 (1983).

22. A. D. Linde, Phys. Lett. B100, 37 (1981).

23. R.-G. Cai, B. Hu and S. Koh, Phys. Lett. B 671, 181 (2009). 
24. K. Marvel and D. Wesley, J. High Energy Phys. 12, 034 (2008).

25. K. Marvel and N. Turok, arXiv:0712.2719.

26. A. R. Brown and E. J. Weinberg, Phys. Rev. D 76, 064003 (2007).

27. J. C. Hackworth and E. J. Weinberg, Phys. Rev. D 71, 044014 (2005).

28. E. J. Weinberg, Phys. Rev. Lett. 98, 251303 (2007).

29. S.-H. H. Tye, D. Wohns and Y. Zhang, arXiv:0811.3753.

30. K. Lee and E. J. Weinberg, Phys. Rev. D 36, 1088 (1987).

31. Y. Kim, K. Maeda and N. Sakai, Nucl. Phys. B 481, 453 (1996).

32. W. Lee, B.-H. Lee, C. H. Lee and C. Park, Phys. Rev. D 74, 123520 (2006)

33. W. Lee, C. Park, B.-H. Lee and C. H. Lee, J. Korean Phys. Soc. 50, S85 (2007).

34. J. Schwinger, Phys. Rev. 82, 664 (1951).

35. S. P. Kim and H. K. Lee, Phys. Rev. D 76, 125002 (2007).

36. D. J. Gross, M. J. Perry and L. G. Yaffe, Phys. Rev. D 25, 330 (1982).

37. P. Ginsparg and M. J. Perry, Nucl. Phys. B 222, 245 (1988).

38. R. R. Caldwell, H. A. Chamblin and G. W. Gibbons, Phys. Rev. D 53, 7103 (1996).

39. B.-H. Lee and W. Lee, arXiv:0809.4907.

40. B.-H. Lee, C. H. Lee, W. Lee and C. Oh, in progress.

41. C. G. Callan and S. Coleman, Phys. Rev. D 16, 1762 (1977).

42. D. Metaxas, arXiv:0804.1045. 